\title{
Zooming in on Field Evaporation Behavior: A Time Depending Density Functional Theory Study
}

\author{
Kaustubh Kaluskar ${ }^{1}$, Joaquin Peralta ${ }^{2}$, Claudia Loyola ${ }^{2}$, Scott Broderick ${ }^{3}$ and Krishna Rajan ${ }^{3}$ \\ 1. Indian Institute of Science Education and Research Bhopal, Department of Chemistry, Bhauri, MP, \\ India \\ 2. Departamento de Ciencias Físicas, Facultad de Ciencias Exactas, Universidad Andrés Bello, Santiago, \\ Chile \\ 3. Department of Materials Design and Innovation, University at Buffalo, Buffalo, NY, USA
}

The evaporation process is a critical phenomenon in Atom Probe Tomography [1] (APT), an experimental technique for studying structure and chemistry of a large variety of materials such as alloys, doped semiconductors, layered materials, geological and biological materials, among others. This approach has emerged as one of the most powerful techniques to analyze semiconductors and device structures at the sub-nanometer scale [2]. APT evaporates atoms on a surface tip by applying a high electric field and a laser pulse, which are reconstructed based on the information of the detector $(\mathrm{X}, \mathrm{Y})$ position and time of flight (tof), giving detailed 3D atomic scale information of the studied structure. Despite the advantages of the technique to provide detailed atomistic information of a sample in real space, there is still a challenge in the reconstruction process [3] of the original sample. Individual ions with their reconstructed coordinates often deviate from the ideal positions and affect the subsequent analysis.

One of the possible reasons for the ion deviation from the ideal positions is the limited understanding of ion trajectory near to the tip surface post field ionization. While ion trajectory away from tip surface can be faithfully modeled considering purely geometrical factors and classical electrodynamics, the near tip surface trajectory can be affected by other causes. Among the main aspects that can bias the trajectory are the neighborhood chemical arrangement [4], laser heating, and electronic effects [5]. To better understand the effects of these factors on the trajectory, we study the near tip trajectory of field evaporated ions for the case of Al-Sc alloy. The electronic effects are modeled using real time - Time Depending Density Functional Theory (RT-TDDFT) and ion movements are modeled using Ehrenfest dynamics. The TDDFT based approach allows us to study the role of electronic effects on trajectory, leading to improved reconstruction algorithms.

In this work, we use both first principle and classical electrodynamics methodologies to produce results of the tip shape evolution and evaporation field values. All calculations were made using the Octupus software [6] to study the evaporation behavior of $\mathrm{Al}-\mathrm{Sc}$ species on $\mathrm{Al}_{3} \mathrm{Sc}$ under two different instrument conditions: (i) laser shape and (ii) energy. Using the RT-TDDFT framework, we identify time and space limits where the ion trajectory is still affected by the specimen. This information will allow us to identify a cutoff in the ion displacement where the effects from surface play an important role in the trajectory. Additionally, based on the velocity and force at the boundary for different initial conditions, we can extrapolate it to differences in the position on the detector. This information can be used to make better informed reconstruction decisions and can be used to assess uncertainty in the reconstruction process in APT experiments [7]. 


\section{References:}

[1] M.K. Miller, and R. G. Forbes. Materials Characterization 60 (2009), p. 461469.

[2] T. F Kelly and D.J. Larson, Annual Review of Materials Research 42 (2012), p.1.

[3] B. Gault et al, Ultramicroscopy, 111 (2011), p. 448.

[4] J. Peralta, S.R. Broderick, and K. Rajan. Ultramicroscopy 132 (2013), p. 143.

[5] C. Loyola et al, J. Vacuum Sci. Tech. A 34 (2016), p. 061404.

[6] X. Andrade et al, Physical Chemistry Chemical Physics 17 (2015), p. 31371.

[7] This work is supported by Proyecto FONDECYT Iniciación 11130501.

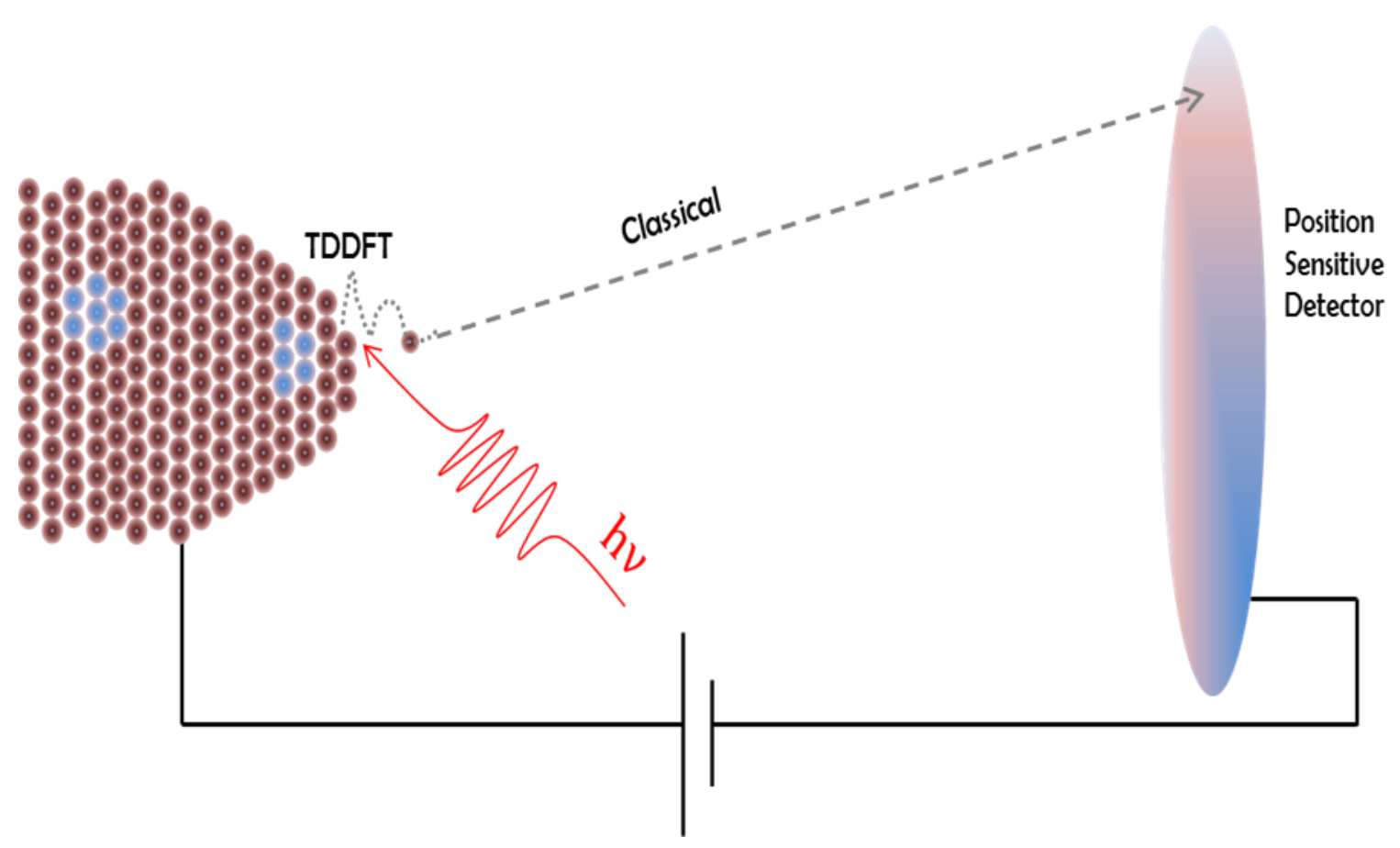

Figure 1. Schematic of the process in an APT experiment. Atoms from the surface are evaporated by the effects of high electric field and laser pulse. At the early stages of the process, the initial displacement of the evaporated atom from the surface can be modeled by using TDDFT, which allows us to understand the effects of different factors in the trajectory of the ions. 